\title{
Mesoscopic Phase Fluctuations: General Phenomenon in Condensed Matter
}

\author{
V.I. Yukalov \\ Bogolubov Laboratory of Theoretical Physics \\ Joint Institute for Nuclear Research, Dubna 141980, Russia
}

\begin{abstract}
General conditions for the occurrence of mesoscopic phase fluctuations in condensed matter are considered. The description of different thermodynamic phases, which coexist as a mixture of mesoscopically separated regions, is based on the theory of heterophase fluctuations. The spaces of states, typical of the related phases, are characterized by weighted Hilbert spaces. Several models illustrate the main features of heterophase condensed matter.
\end{abstract}

PACS numbers: 05.40.-a, 05.70.Ce, 05.70.Fh, 05.70.Jk, 64.60.-i

Keywords: Fluctuation phenomena; Equations of state; Phase transitions; Critical phenomena 


\section{Introduction}

In many cases, condensed matter does not consist of a unique thermodynamic phase but is separated into mesoscopic regions filled by different phases. A number of examples of such matter are described in review [1]. The coexisting phases can be distinguished by their order parameters that change from one mesoscopic region to another. The order parameters are often associated with some special symmetry [2]. However, this is not compulsory, and in many cases different phases cannot be characterized by different kinds of symmetry. For instance, crystalline and fluid phases have different spatial symmetry. Magnetic and paramagnetic phases possess different magnetic symmetry, which can be observed by means of neutron scattering [3]. Similarly, ferroelectric and paraelectric phases possess different symmetry characterized by polarization. If the latter phases coexist, the polarization of the mixed system is spatially nonuniform [4,5]. Contrary to this, liquid and gas cannot be distinguished by their symmetry, though they may have different densities or other properties [6]. Metal and nonmetal are also distinguished not by symmetry but by their conductivity. Better and worse conducting mesoscopic regions can coexist in many materials [7-9].

Mesoscopic is a key word here, implying the following. Suppose that inside one thermodynamic phase there appear the nuclei of another phase. Each nucleus, in order to represent a coherent germ of a phase, should have a characteristic coherence length $l_{\text {coh }}$ much longer than the mean interparticle distance $a$. As is evident, an object of the size $a$ cannot be associated with a thermodynamic phase. At the same time, the coherence length is to be essentially shorter than the characteristic length $L$ of the whole system. Thus, the phase germs are of the mesoscopic size [1], that is

$$
a \ll l_{\text {coh }} \ll L \text {. }
$$

This property is to be understood on the average. Generally, the shape of heterophase germs is not necessarily regular, but can be rather complicated. Several germs can also touch each other forming very ramified fractal-type objects, similar to fractal animals at criticality [10]. The total effective length of such a fractal formation can reach the length $L$ of the whole system. Being mesoscopic in size, the heterophase nuclei are randomly distributed in space, forming no regular structures.

Random is another key word. Heterophase germs are random in space, analogously to random disordering defects in solids $[11,12]$, but being different from the latter by the mesoscopic size of the phase germs.

These germs, being mesoscopic in space, can also be mesoscopic in time. This means that they may be not static but dynamical, arising and then disappearing, with a characteristic lifetime, or coherence time $t_{c o h}$, such that

$$
\tau_{l o c} \ll t_{c o h} \ll T_{o b s},
$$

where $\tau_{l o c}$ is the time of local equilibrium and $T_{o b s}$ is an observation time [1]. The temporal behaviour of these fluctuational dynamic germs is chaotic. When the system is chaotic in time and random in space, it is termed turbulent. Since here the spatio-temporal chaoticity is connected with the dynamics of heterophase nuclei, this phenomenon may be called heterophase turbulence [13]. The origin of heterophase turbulence can be classified into three types, extrinsic, intrinsic, and stochastic [13]. 
Extrinsic origin of turbulence assumes the existence of sufficiently strong external forces

acting on the system. Such forces can induce chaoticity in classical and quasiclassical systems $[14,15]$.

Intrinsic origin presupposes that the system behaves chaotically owing to its own internal properties. There are many examples of such chaotic dynamical systems [16,17]. For statistical systems this means that quasiequilibrium heterophase states are their chaotic attractors $[1,13,18]$.

Stochastic origin of heterophase turbulence is intermediate between the above two. It takes into account that all real systems are never completely isolated but can be only quasi-isolated, and that an infinitesimally small external stochastic perturbation can trigger chaoticity, making the system stochastically unstable $[1,18,19]$.

The most interesting cases are those related to the intrinsic and stochastic origins, when heterophase states are not forced by external sources but are self-organized. There exist plenty of examples of such systems, reviewed in Ref. 1. Among those heterophase materials that have attracted much attention in recent days, it is possible to mention liquid crystals [20], high-temperature superconductors [21-24] and colossal magnetoresistant materials [25]. Mesoscopic phase fluctuations lead to the occurrence of many unusual features of matter, for instance such as colossal magnetoresistence [25] and anomalous diffusion in solids [26]. Their appearance may transform a second-order phase transition into a first-order one [1]. They can induce different anomalies in the behaviour of thermodynamic and dynamic characteristics of matter, for instance: the maxima of specific heat below the critical temperature [27], specificheat jumps at the nucleation point [1,28], anomalous saggings of the Mössbauer factor at the phase transition point [29,30], anomalous decrease of sound velocity and increase of isothermal compressibility at the point of a structural transition $[29,30]$, and other anomalies [1].

All unusual features of condensed matter with mesoscopic phase fluctuations can be selfconsistently described in the frame of the statistical theory of heterophase fluctuations [1]. In order not to force readers to study the whole review [1], a brief survey of this theory is presented in Sections 2 and 3. The main purpose of this presentation is, from one side, to give a rather short account of the principal ideas, the theory is based on, and from another side, to clearly point at the principal mathematical techniques providing the foundation. An attempt of combining brevity with accuracy in outlining the theory is a methodical novelty of the paper. Several examples of sections 4 to 6 illustrate applications of the theory. The generality of the approach to treating mesoscopic phase fluctuations as well as the generality of the phenomenon itself are emphasized in Section 7.

\section{Description of Phase Separation}

When talking about phase separation, one, first of all, keeps in mind the separation of thermodynamic phases in real space. Different phases can be distinguished by their different properties. Those phase characteristics that are the most appropriate for distinguishing different phases are called the order parameters. The order parameter of a thermodynamic phase is a set $\eta$ of quantities typical of the phase and uniquely distinguishing it from other 
phases. If there are several phases, each of them is characterized by the corresponding order parameter $\eta_{\nu}$, with the index $\nu=1,2, \ldots$ enumerating the phases. When a physical system, occupying a real-space volume $\mathbb{V}$, is phase separated, then this volume can be divided into subvolumes $\mathbb{V}_{\nu}$ filled by different phases with different order parameters $\eta_{\nu}$. A family $\left\{\mathbb{V}_{\nu}\right\}$ of subvolumes $\mathbb{V}_{\nu}$ forms an orthogonal covering of $\mathbb{V}$, such that

$$
\mathbb{V}=\bigcup_{\nu} \mathbb{V}_{\nu}, \quad \mathbb{V}_{\mu} \bigcap \mathbb{V}_{\nu}=\delta_{\mu \nu} \mathbb{V}_{\nu}
$$

The latter can be represented as the resolution of unity

$$
\sum_{\nu} \xi_{\nu}(\mathbf{r})=1 \quad(\mathbf{r} \in \mathbb{V})
$$

for the manifold indicator functions

$$
\xi_{\nu}(\mathbf{r})= \begin{cases}1, & \mathbf{r} \in \mathbb{V}_{\nu} \\ 0, & \mathbf{r} \notin \mathbb{V}_{\nu}\end{cases}
$$

A family of indicator functions

$$
\xi \equiv\left\{\xi_{\nu}(\mathbf{r}) \mid \nu=1,2, \ldots ; \mathbf{r} \in \mathbb{V}\right\}
$$

uniquely defines a phase configuration in the real-space volume $\mathbb{V}$.

In physical systems, the separation of thermodynamic phases is usually realized so that these phases are not sharply split apart, but are transformed one into another through an interphase layer, where the order parameter continuously changes from the value typical of one phase to that characteristic of another phase. How could we then locate a separating surface between such phases? First, the location of the separating surface can be fixed in a unique manner by employing the Gibbs method of separating surfaces [31]. This can be done by invoking some additional conditions. For instance, the equimolecular separating surface is uniquely defined by requiring that the surface density of matter be zero. Moreover, a precise location of the separating surface is not important. This is because the phase configuration in what follows will be varied, with the family of indicator functions (3) being treated as a random variable.

In order to define a method of varying phase configurations, we should consider a manifold $\{\xi\}$ of all possible families (3) and to rig this manifold with a differential measure. To this end, we introduce an orthogonal subcovering $\left\{\mathbb{V}_{\nu i}\right\}$ of the volume $\mathbb{V}_{\nu}$, so that

$$
\mathbb{V}_{\nu}=\bigcup_{i=1}^{n_{\nu}} \mathbb{V}_{\nu i}, \quad \mathbb{V}_{\mu i} \bigcap \mathbb{V}_{\nu j}=\delta_{\mu \nu} \delta_{i j} \mathbb{V}_{\nu i}
$$

To each subregion $\mathbb{V}_{\nu i}$, we ascribe a vector $\mathbf{a}_{\nu i} \in \mathbb{V}_{\nu i}$ playing the role of a local center of coordinates, so that moving $\mathbf{a}_{\nu i}$ implies a congruent motion of $\mathbb{V}_{\nu i}$. The shapes of the subvolumes $\mathbb{V}_{\nu i}$ as well as the location of the centers $\mathbf{a}_{\nu i}$ inside each $\mathbb{V}_{\nu i}$ can be arbitrary. For a given subcovering (4), the indicator functions (2) can be decomposed as

$$
\xi_{\nu}(\mathbf{r})=\sum_{i=1}^{n_{\nu}} \xi_{\nu i}\left(\mathbf{r}-\mathbf{a}_{\nu i}\right)
$$


where

$$
\xi_{\nu i}\left(\mathbf{r}-\mathbf{a}_{\nu i}\right)= \begin{cases}1, & \mathbf{r} \in \mathbb{V}_{\nu i} \\ 0, & \mathbf{r} \notin \mathbb{V}_{\nu i}\end{cases}
$$

The differential measure on the manifold $\{\xi\}$ is introduced [1] as the differential functional measure

$$
\mathcal{D} \xi \equiv \lim _{\left\{n_{\nu} \rightarrow \infty\right\}} \delta\left(\sum_{\nu} x_{\nu}-1\right) \prod_{\nu} d x_{\nu} \prod_{\nu} \prod_{i=1}^{n_{\nu}} \frac{d \mathbf{a}_{\nu i}}{V}
$$

in which

$$
x_{\nu} \equiv \frac{1}{V} \int_{\mathbb{V}} \xi_{\nu}(\mathbf{r}) d \mathbf{r}, \quad V \equiv \int_{\mathbb{V}} d \mathbf{r} .
$$

The measure (6), with varying $x_{\nu} \in[0,1]$ and $\mathbf{a}_{\nu i} \in \mathbb{V}$, induces a topology on the manifold $\{\xi\}$. This results in the topological configuration space

$$
\mathcal{X} \equiv\{\xi \mid \mathcal{D} \xi\}
$$

composed of all possible phase configurations.

There exists another principal problem connected with phase separation. This is how such a macroscopic quantity as a thermodynamic phase is related to the microscopic states of a physical system. For generality, we consider this question for quantum systems.

Suppose that all admissible microscopic quantum states of a physical system form a Hilbert space $\mathcal{H}$. Let $\left\{\varphi_{n}\right\}$ be an orthonormal basis in this space, such that $\left(\varphi_{m}, \varphi_{n}\right)=\delta_{m n}$. Then the space of microstates can be presented as a closed linear envelope

$$
\mathcal{H} \equiv \overline{\mathcal{L}}\left\{\varphi_{n}\right\}
$$

over the basis $\left\{\varphi_{n}\right\}$. Any state $f \in \mathcal{H}$ can be written as a decomposition

$$
f=\sum_{n} f_{n} \varphi_{n}, \quad f_{n} \equiv\left(\varphi_{n}, f\right) .
$$

For any two states $f, f^{\prime} \in \mathcal{H}$, their scalar product is given by the equation

$$
\left(f, f^{\prime}\right)=\sum_{n} f_{n}^{*} f_{n}^{\prime}
$$

Now assume that for each representative $\varphi_{n}$ of the basis $\left\{\varphi_{n}\right\}$ a weight $p_{\nu}\left(\varphi_{n}\right)$ is assigned, normalized so that

$$
\sum_{n} p_{\nu}\left(\varphi_{n}\right)=1
$$

This defines the weighted basis $\left\{\varphi_{n}, p_{\nu}\left(\varphi_{n}\right)\right\}$. The weighted scalar product is introduced as

$$
\left(f, f^{\prime}\right)_{\nu} \equiv \sum_{n} p_{\nu}\left(\varphi_{n}\right) f_{n}^{*} f_{n}^{\prime}
$$

Then a closed linear envelope over the weighted basis results [1] in the weighted Hilbert space

$$
\mathcal{H}_{\nu} \equiv \overline{\mathcal{L}}\left\{\varphi_{n}, p_{\nu}\left(\varphi_{n}\right)\right\},
$$


with the associated scalar product (9).

From definition (9), one has

$$
\left(\varphi_{m}, \varphi_{n}\right)_{\nu}=p_{\nu}\left(\varphi_{n}\right) \delta_{m n}, \quad\left(\varphi_{n}, f\right)_{\nu}=p_{\nu}\left(\varphi_{n}\right)\left(\varphi_{n}, f\right)
$$

For an operator $A_{\nu}$, given on $\mathcal{H}_{\nu}$, the trace operator is defined as

$$
\operatorname{Tr}_{\mathcal{H}_{\nu}} A_{\nu} \equiv \sum_{n}\left(\varphi_{n}, A_{\nu} \varphi_{n}\right)_{\nu}
$$

From equalities (11), it follows that

$$
\left(\varphi_{m}, A_{\nu} \varphi_{n}\right)_{\nu}=p_{\nu}\left(\varphi_{m}\right)\left(\varphi_{m}, A_{\nu} \varphi_{n}\right)
$$

Therefore the trace (12) reads

$$
\operatorname{Tr}_{\mathcal{H}_{\nu}} A_{\nu} \equiv \sum_{n} p_{\nu}\left(\varphi_{n}\right)\left(\varphi_{n}, A_{\nu} \varphi_{n}\right)
$$

Using the above formulas, we may specify the weighted average

$$
<A_{\nu}>\equiv \operatorname{Tr}_{\mathcal{H}_{\nu}} \hat{\rho}_{\nu} A_{\nu}
$$

of an operator $A_{\nu}$, with $\hat{\rho}_{\nu}$ being a statistical operator on $\mathcal{H}_{\nu}$, normalized so that

$$
\operatorname{Tr}_{\mathcal{H}_{\nu}} \hat{\rho}_{\nu}=1
$$

The order parameters $\eta_{\nu}$ can be presented as the averages

$$
\eta_{\nu} \equiv<\hat{\eta}_{\nu}>
$$

of the corresponding order operators $\hat{\eta}_{\nu}$. Each order parameter is assumed to possess the properties classifying the related thermodynamic phase. These properties implicitly determine the appropriate basis weight $p_{\nu}\left(\varphi_{n}\right)$. The latter is not uniquely defined by Eq. (15), but, generally, there can exist a class of such basis weights satisfying the same Eq. (15). Any representative of this class is acceptable, provided all of them yield the coinciding averages. In practice, it is even not necessary to find an explicit form of $p_{\nu}\left(\varphi_{n}\right)$, but it is sufficient to impose the appropriate restrictions on the averages, which would guarantee the typical features of the related phases [1]. There are several ways of selecting pure phases, such as the Bogolubov method of infinitesimal sources [32], the method of thermodynamic quasiaverages $[1,33]$, the method of order indices [34,35], and others [1]. A very convenient way is by imposing additional conditions on density matrices or Green functions [36]. A similar way of imposing conditions on distributions or correlation functions is also suitable for classical systems. 


\section{Heterophase Statistical State}

The total space of microstates for a system with phase separation is the weighted fiber space

$$
\mathcal{Y} \equiv \otimes_{\nu} \mathcal{H}_{\nu}
$$

whose fibering yields the weighted Hilbert spaces (10).

Let $A_{\nu}\left(\xi_{\nu}\right)$ be an operator on $\mathcal{H}_{\nu}$, when a phase configuration (3) is fixed. One may also consider the operator density $A_{\nu}\left(\xi_{\nu}, \mathbf{r}\right)$ for which

$$
A_{\nu}\left(\xi_{\nu}\right)=\int A_{\nu}\left(\xi_{\nu}, \mathbf{r}\right) d \mathbf{r}
$$

Here and in what follows, the integration with respect to $\mathbf{r}$, if not specified, implies the integration over the whole system volume $\mathbb{V}$. The operator density on the total space (16) is defined as

$$
A(\xi, \mathbf{r}) \equiv \oplus_{\nu} A_{\nu}\left(\xi_{\nu}, \mathbf{r}\right)
$$

so that the related operator is

$$
A(\xi) \equiv \int A(\xi, \mathbf{r}) d \mathbf{r}=\oplus_{\nu} A_{\nu}\left(\xi_{\nu}\right)
$$

Examples of operator densities are the Hamiltonian density $H(\xi, \mathbf{r})$ and the number-ofparticle operator density $N(\xi, \mathbf{r})$.

Statistical properties of a heterophase physical system, with a fixed phase configuration, can be characterized by the quasiequilibrium Gibbs ensemble [1]. A particular phase configuration $\xi$ is connected with the local inverse temperature $\beta(\xi, \mathbf{r})$ and local chemical potential $\mu(\xi, \mathbf{r})$. The latter enter in the quasi-Hamiltonian

$$
Q(\xi) \equiv \int \beta(\xi, \mathbf{r})[H(\xi, \mathbf{r})-\mu(\xi, \mathbf{r}) N(\xi, \mathbf{r})] d \mathbf{r}
$$

This defines the quasiequilibrium thermodynamic potential, which will be called quasipotential

$$
q \equiv-\frac{1}{N} \ln \operatorname{Tr}_{\mathcal{Y}} \int e^{-Q(\xi)} \mathcal{D} \xi
$$

with $N$ being the total number of particles and where the integration with respect to $\xi$ is over the configuration space (7) of all possible phase configurations. The quasiequiblibrium statistical operator is

$$
\hat{\rho}(\xi) \equiv \frac{\exp \{-Q(\xi)\}}{\operatorname{Tr}_{\mathcal{Y}} \int \exp \{-Q(\xi)\} \mathcal{D} \xi} .
$$

Expectation values of operators $A(\xi)$ are given by the averages

$$
<\tilde{A}>\equiv \operatorname{Tr}_{\mathcal{Y}} \int \hat{\rho}(\xi) A(\xi) \mathcal{D} \xi
$$

Integrating with respect to phase configurations, we assume that measuring procedures effectively average the observable quantities over random phase configurations. If all parts 
of the heterophase system ate locally equivalent to each other, then the local inverse temperature and local chemical potential, being averaged over phase configurations, should not depend on $\mathbf{r}$, which can be formulated as the condition of heterophase equilibrium

$$
\beta=\int \beta(\xi, \mathbf{r}) \mathcal{D} \xi, \quad \mu=\int \mu(\xi, \mathbf{r}) \mathcal{D} \xi
$$

Accomplishing the integration over the configuration space (7), we keep in mind the existence of the thermodynamic limit

$$
N \rightarrow \infty, \quad V \rightarrow \infty, \quad \frac{N}{V} \rightarrow \text { const } .
$$

Then the thermodynamic quasipotential (21) reduces [1] to

$$
q=-\frac{1}{N} \ln \operatorname{Tr}_{\mathcal{Y}} e^{-\beta \tilde{H}}
$$

with the effective Hamiltonian

$$
\tilde{H}=\oplus_{\nu} H_{\nu}, \quad H_{\nu}=H_{\nu}\left(w_{\nu}\right) .
$$

The terms

$$
H_{\nu}=\int\left[H_{\nu}\left(w_{\nu}, \mathbf{r}\right)-\mu N_{\nu}\left(w_{\nu}, \mathbf{r}\right)\right] d \mathbf{r}
$$

of the Hamiltonian (26) can be called [1] the phase-replica Hamiltonians. The operator densities $A_{\nu}\left(w_{\nu}, \mathbf{r}\right)$ have the same form as $A_{\nu}\left(\xi_{\nu}, \mathbf{r}\right)$, but with $\xi_{\nu}$ replaced by $w_{\nu}$. The quantities $w_{\nu}$ are the phase probabilities, playing the role of average geometric probabilities for the corresponding phases. These probabilities are defined [1] as the minimizers of the thermodynamic quasipotential (25), that is,

$$
q=\operatorname{abs} \min _{\left\{w_{\nu}\right\}} q\left(\left\{w_{\nu}\right\}\right)
$$

under the condition

$$
\sum_{\nu} w_{\nu}=1, \quad 0 \leq w_{\nu} \leq 1
$$

For the operator averages (23), we find [1]

$$
<\tilde{A}>=\operatorname{Tr}_{\mathcal{Y}} \hat{\rho} \tilde{A}
$$

where the effective statistical operator

$$
\hat{\rho} \equiv \frac{\exp (-\beta \tilde{H})}{\operatorname{Tr}_{\mathcal{Y}} \exp (-\beta \tilde{H})},
$$

is expressed through the Hamiltonian (26). The operator $\tilde{A}$ in the right-hand side of Eq. (29) is

$$
\tilde{A} \equiv \oplus_{\nu} A_{\nu}, \quad A_{\nu}=A_{\nu}\left(w_{\nu}\right)
$$


From Eqs. (26) and (30) it follows that

$$
\hat{\rho}=\otimes_{\nu} \hat{\rho}_{\nu}, \quad \hat{\rho}_{\nu} \equiv \frac{\exp \left(-\beta H_{\nu}\right)}{\operatorname{Tr}_{\mathcal{H}_{\nu}} \exp \left(-\beta H_{\nu}\right)} .
$$

Therefore the average (29) can be written as

$$
<\tilde{A}>=\sum_{\nu}<A_{\nu}>
$$

The set $\{\tilde{A}\}$ of all operators, corresponding to observable quantities, composes an algebra of local observables $\tilde{\mathcal{A}}$. The heterophase statistical state is the family $<\tilde{\mathcal{A}}>$.

The operators from the algebra of local observables can be divided into single-particle, two-particle, and, generally, $k$-particle operators, so that the overall structure of an operator $A_{\nu}$ is

$$
A_{\nu}=\sum_{k} \frac{1}{k !} w_{\nu}^{k} A_{\nu}^{(k)}
$$

The standard Hamiltonians of statistical systems usually contain the single-particle and the two-particle terms only. Hence the structure of a replica Hamiltonian is

$$
H_{\nu}=w_{\nu} H_{\nu}^{(1)}+\frac{1}{2} w_{\nu}^{2} H_{\nu}^{(2)}
$$

Assuming that $H_{\nu}^{(k)}$ do not depend on $w_{\nu}$, we may explicitly derive the conditions of heterophase stability.

Consider, for concreteness, a heterophase mixture of two phases. It is convenient to introduce the notation

$$
w \equiv w_{1}, \quad w_{2}=1-w .
$$

Then the phase probability $w$ is a minimizer of the thermodynamic quasipotential $q=q(w)$, which implies the equations

$$
\frac{\partial q}{\partial w}=0, \quad \frac{\partial^{2} q}{\partial w^{2}}>0
$$

The first of the latter, taking into account form (25), yields

$$
<\frac{\partial \tilde{H}}{\partial w}>=0
$$

The second of Eqs. (37) has the meaning of the positive definiteness of the heterophase susceptibility

$$
\tilde{\chi} \equiv \frac{\partial^{2} q}{\partial w^{2}}>0
$$

Taking account of Eq. (25), we have

$$
\tilde{\chi}=\frac{\beta}{N}\left[<\frac{\partial^{2} \tilde{H}}{\partial w^{2}}>-\beta<\left(\frac{\partial \tilde{H}}{\partial w}\right)^{2}>\right] .
$$


Then the stability condition (39) reads

$$
\left(<\frac{\partial^{2} \tilde{H}}{\partial w^{2}}>\right)>\beta<\left(\frac{\partial \tilde{H}}{\partial w}\right)^{2}>
$$

Introducing the averages

$$
K_{\nu} \equiv \frac{1}{N}<H_{\nu}^{(1)}>, \quad \Phi_{\nu} \equiv \frac{1}{N}<H_{\nu}^{(2)}>
$$

we find

$$
\frac{1}{N}<\frac{\partial \tilde{H}}{\partial w}>K_{1}+w \Phi_{1}-K_{2}-(1-w) \Phi_{2}, \quad \frac{1}{N}<\frac{\partial^{2} \tilde{H}}{\partial w^{2}}>=\Phi_{1}+\Phi_{2} .
$$

The extremum condition (38) yields an equation for the phase probability

$$
w=\frac{\Phi_{2}+K_{2}-K_{1}}{\Phi_{1}+\Phi_{2}}
$$

From the inequalities $0 \leq w \leq 1$ it follows that

$$
-\Phi_{1} \leq K_{1}-K_{2} \leq \Phi_{2}
$$

And the stability condition (41) gives

$$
\Phi_{1}+\Phi_{2}>\frac{\beta}{N}\left[<\left(\frac{\partial \tilde{H}}{\partial w}\right)^{2}>\right]>0 .
$$

Inequalities (44) and (45) can be called the conditions of heterophase stability. These are to be complimented by the usual conditions of thermodynamic stability, such as the positivity of specific heat and of isothermal compressibility.

\section{Theory of Melting and Crystallization}

An important application of the developed approach is to describing the phase transitions of melting and crystallization $[37,38]$. The mesoscopic fluctuations inside a crystal are the liquid-like droplets presented by the regions of local disorder of the crystalline lattice, arising below melting temperature. Above the latter, mesoscopic heterophase fluctuations are quasicrystalline clusters inside liquid.

To consider this phase transition, let us take the replica Hamiltonian in the standard form

$$
\begin{gathered}
H_{\nu}=w_{\nu} \int \psi_{\nu}^{\dagger}(\mathbf{r})\left(-\frac{\hbar^{2} \nabla^{2}}{2 m_{0}}-\mu\right) \psi_{\nu}(\mathbf{r}) d \mathbf{r}+ \\
+\frac{1}{2} w_{\nu}^{2} \int \psi_{\nu}^{\dagger}(\mathbf{r}) \psi_{\nu}^{\dagger}\left(\mathbf{r}^{\prime}\right) \Phi\left(\mathbf{r}-\mathbf{r}^{\prime}\right) \psi_{\nu}\left(\mathbf{r}^{\prime}\right) \psi_{\nu}(\mathbf{r}) d \mathbf{r} d \mathbf{r}^{\prime},
\end{gathered}
$$


in which $\psi_{\nu}(\mathbf{r})$ are field operators. The chemical potential $\mu$ can be defined from the equation

$$
N=N_{1}+N_{2}, \quad N_{\nu} \equiv-<\frac{\partial H_{\nu}}{\partial \mu}>
$$

Here, we deal with a two-phase mixture, because of which $\nu=1,2$.

The local density of particles in a $\nu$-phase is

$$
\rho_{\nu}(\mathbf{r}) \equiv<\psi_{\nu}^{\dagger}(\mathbf{r}) \psi_{\nu}(\mathbf{r})>
$$

The number of particles in the $\nu$-phase, according to the definition in Eq. (47), becomes

$$
N_{\nu}=w_{\nu} \int \rho_{\nu}(\mathbf{r}) d \mathbf{r}
$$

The average density of the $\nu$-phase is

$$
\rho_{\nu}=\frac{1}{V} \int \rho_{\nu}(\mathbf{r}) d \mathbf{r}
$$

And the mean density of particles in the whole system writes

$$
\rho \equiv \frac{N}{V}=w_{1} \rho_{1}+w_{2} \rho_{2}
$$

If the density $\rho$ is fixed, then the latter equation defines the chemical potential $\mu=\mu(\rho, T)$ as a function of density and temperature.

Let the crystalline phase be numbered with $\nu=1$, while the liquid phase, with $\nu=2$. By this assumption, the local density (48) for the crystalline phase is periodic,

$$
\rho_{1}(\mathbf{r}+\mathbf{a})=\rho_{a}(\mathbf{r}) \neq \text { const },
$$

where $\mathbf{a}$ is any lattice vector. But the liquid phase has a uniform density

$$
\rho_{2}(\mathbf{r})=\rho_{2}(0)=\text { const }
$$

Since it is the local density that distinguishes the phases, $\rho_{\nu}(\mathbf{r})$ can be treated as the order parameter. Alternatively, it is possible to define the order parameter

$$
\eta_{\nu} \equiv \sup _{\mathbf{r}} \frac{\rho_{\nu}(\mathbf{r})}{\rho_{\nu}}-1
$$

For an ideal crystal, $\sup _{\mathbf{r}} \rho_{1}(\mathbf{r})=\rho_{1}(\mathbf{a})$, but for a liquid, $\rho_{2}(\mathbf{r})=$ const. Therefore

$$
\eta_{1}=\frac{\rho_{1}(\mathbf{a})}{\rho_{1}}-1>0, \quad \eta_{2} \equiv 0 .
$$

The analysis $[37,38]$ of the typical behaviour of the phase probabilities $w_{\nu}$ results in the following figures. In Fig. 1, the probability of the crystalline phase is shown as a function of temperature $\Theta$ in arbitrary units. At zero temperature, the system is in pure crystalline 
phase. For higher temperatures, the crystalline-phase probability slightly diminishes along the line $\mathbf{A B}$ and then abruptly falls down from the point $\mathbf{B}$ to $\mathbf{E}$ at the melting temperature $\Theta_{m}$. Above the latter, the curve $\mathbf{E F}$ corresponds to the probability of crystalline clusters inside the liquid phase. The branches $\mathbf{B C}$ and $\mathbf{D E}$ are related to metastable states of an overheated crystal and crystalline clusters inside an overcooled liquid, respectively.

Figure 2 displays the probability of the liquid phase $w_{2}=1-w_{1}$. At zero temperature, $w_{2}=0$. With increasing temperature, there appear a small probability of liquid-like disordered regions in the crystalline phase, which rises along the line ab. At the melting temperature $\Theta_{m}$, the probability of the liquid phase jumps up to the point $\mathbf{e}$, after which it follows the line ef. The branches bc and de again are related to metastable states.

In the similar way, one may treat a liquid-glass transition. A glassy state can be described by a disordered lattice. Then the local density $\rho_{1}(\mathbf{r})$ is not periodic, but, at the same time, it is not constant. The glassy solid state and liquid state can be distinguished by the order parameter (54), so that, similarly to Eqs. (55), one has

$$
\eta_{1}>0, \quad \eta_{2}=0
$$

Possible behaviour of the glassy-phase probability is shown in Fig. 3. Contrary to the crystalliquid transition, the glass-liquid transition can occur as a continuous transition, without the jumps of phase probabilities at the glassyfication temperature $\Theta_{g}$. The glassy state is metastable, being not an absolute extremum of a thermodynamic potential, as compared to the crystalline state.

Thermodynamic potentials for heterophase matter are the same as for pure-phase systems. We can consider, for instance, the grand potential

$$
\Omega=-p V=\Omega(T, V, \mu),
$$

the free energy

$$
F=\Omega+\mu N=F(T, V, N),
$$

or the Kramers potential

$$
K=-\frac{\Omega}{T}=K(\beta, V, \beta \mu) .
$$

All of them can be expressed through the quasipotential (25) by means of the equations for the grand potential

$$
\Omega=N q T,
$$

free energy

$$
F=N(q T+\mu),
$$

or for the Kramers potential

$$
K=-N q .
$$

As is seen from the latter relation, the quasipotential (25) is minus the Kramers potential per particle. From the above equations, the pressure is

$$
p=-\frac{\Omega}{V}=-\rho q T,
$$


where $\rho$ is the mean density (51). The minimum of the quasipotential (25) corresponds to that of the grand potential (67) or to the minimum of the free energy (58), but to the maximum of the Kramers potential (59).

The quasipotential (25), due to the form of the effective Hamiltonian (26), can be written as a sum

$$
q=\sum_{\nu} q_{\nu}, \quad q_{\nu} \equiv-\frac{1}{N} \ln \operatorname{Tr}_{\mathcal{H}_{\nu}} e^{-\beta H_{\nu}}
$$

Thence, the grand potential (57) becomes

$$
\Omega=\sum_{\nu} \Omega_{\nu}, \quad \Omega_{\nu} \equiv-T \ln \operatorname{Tr}_{\mathcal{H}_{\nu}} e^{-\beta H_{\nu}} .
$$

And the free energy (58) also writes as a sum

$$
F=\sum_{\nu} F_{\nu}, \quad F_{\nu} \equiv \Omega_{\nu}+\mu N_{\nu}
$$

However, one should not forget that the terms in the sums (61) to (63) depend on thermodynamic parameters as well as on the phase probabilities, e.g.,

$$
\Omega_{\nu}=\Omega_{\nu}\left(T, V, \mu, w_{\nu}\right), \quad F_{\nu}=F_{\nu}\left(T, V, N, w_{\nu}\right)
$$

where $w_{\nu}$ is a function of $T, V, \mu$ or $T, V, N$, respectively. These terms correspond to phase replicas of a heterophase system, but not to pure phases. The latter would be described by the potentials

$$
\Omega_{\nu}^{\prime}=\Omega_{\nu}^{\prime}\left(T, V_{\nu}, \mu\right)=\Omega_{\nu}\left(T, V_{\nu}, \mu, 1\right), \quad F_{\nu}^{\prime}=F_{\nu}^{\prime}\left(T, V_{\nu}, N_{\nu}\right)=F_{\nu}\left(T, V_{\nu}, N_{\nu}, 1\right) .
$$

These do not compose the thermodynamic potentials (62) and (63) of a heterophase system, that is,

$$
\Omega \neq \sum_{\nu} \Omega_{\nu}^{\prime}, \quad F \neq \sum_{\nu} F_{\nu}^{\prime}
$$

The difference

$$
\Omega_{\text {int }} \equiv \Omega-\sum_{\nu} \Omega_{\nu}^{\prime}, \quad F_{\text {int }} \equiv F-\sum_{\nu} F_{\nu}^{\prime}
$$

between the total thermodynamic potentials (62) or (63) and the sums of the related potentials (65), representing pure phases, can be associated with the thermodynamic potentials of interphase layers [1]. Analogous conclusions concern the Kramers potential (59), for which

$$
K=\sum_{\nu} \ln \operatorname{Tr}_{\mathcal{H}_{\nu}} e^{-\beta H_{\nu}}
$$

But this potential is a function $K(\beta, V, \beta \mu)$ of not the most convenient thermodynamic variables, because of which it is rarely employed in practice.

In this way, the whole heterophase system is characterized by a total thermodynamic potential, like the grand potential (62) or free energy (63). Such a potential writes as a sum of the terms representing phase replicas of heterophase matter, so that these terms should not be confused with the potentials of pure phases. A phase-transition point, defined in 
the theory of heterophase fluctuations, can be rather different from a transition point given by the conventional approach dealing solely with pure systems. In the latter case, a phase transition between two phases, say the melting-crystallization transition, is defined by the equalities

$$
\Omega_{1}^{\prime}(T, V, \mu)=\Omega_{2}^{\prime}(T, V, \mu), \quad F_{1}^{\prime}(T, V, N)=F_{2}^{\prime}(T, V, N)
$$

for the potentials of pure phases, which give, e.g., the transition temperature $T^{\prime}(\mu)$ or $T^{\prime}(\rho)$, respectively. This temperature coincides with the transition temperature $T_{0}$ in the theory of heterophase matter only if $w_{1} \equiv 1$ and $w_{2} \equiv 0$ below $T_{0}$, while $w_{1} \equiv 0, w_{2} \equiv 1$ above $T_{0}$. However, generally, $w_{\nu} \not \equiv 0,1$, and the transition point in heterophase matter is different from that in pure-phase systems. Thus, the phase transition temperature $T_{0}$ in heterophase matter is defined as the point where the relation between $w_{1}$ and $w_{2}$ changes so that

$$
w_{1}>w_{2} \quad\left(T=T_{0}-0\right), \quad w_{1}<w_{2} \quad\left(T=T_{0}+0\right) .
$$

In addition, the existence of mesoscopic heterophase fluctuations may lead to various pretransitional effects [1], which can be explained only taking these fluctuations into account.

\section{Turbulent versus Chaotic Crystals}

The majority of crystals are associated with a periodic crystalline structure. There exist as well quasicrystals, characterized by a quasiperiodic spatial distribution of particles [39]. Crystals and quasicrystals can form stable matter. The structure that is not uniform and neither periodic nor quasiperiodic, can be called chaotic. Examples of solids with chaotic structure are glasses and amorphous materials. In nature, such chaotic solids are metastable. Ruelle [40] put forward a question asking if solids with random spatial structure could form not metastable but stable states. Newell and Pomeau [41] mentioned that such static chaotic spatial structures could exist in principle, which was supported by a two-dimensional numerical modeling [42]. The solids with chaotic spatial structures can be named chaotic crystals. Sometimes, they are also termed as turbulent, which, however, is clearly a misnomer. Turbulence is a spatio-temporal phenomenon characterizing unstable temporal evolution of chaotic spatial structures [43]. It is, therefore, incorrect to call a static spatial structure turbulent. To dignify a phenomenon by the name of turbulence compulsory implies the involvement of chaotic temporal evolution. Therefore stable solids with a static random spatial structure [40-42] can be correctly classified only as chaotic crystals.

The existence of temporal heterophase fluctuations is what can render solids really turbulent. Randomly fluctuating in time and chaotically distributed in space mesoscopic heterophase fluctuations realize heterophase turbulence [13]. A stable solid exhibiting heterophase turbulence is a turbulent crystal. The averaging over heterophase configurations, described is Section 3, leads to an effective quasiequilibrium picture of heterophase turbulent matter, similarly to the averaged treatment of usual turbulence [43].

Heterophase turbulence may develop in any condensed matter. Moreover, it is heterophase turbulence which is the dynamical cause of all phase transitions. The melting-crystallization transition, discussed in Section 4 is not an exception. Heterophase turbulence in a crystal shows up as chaotically appearing and disappearing germs of liquid-like regions of disordered 
lattice. The crystalline and fluid phases can be distinguished between each other by their differing symmetries resulting in different features of their spatial local densities (52) and (53). Competing phases quite often differ in their symmetry, which, though, is not a mandatory prerequisite for their distinction. Thermodynamic phases may, for instance, having the same symmetry, possess different mean densities, as liquid and gas. The possibility of heterophase turbulence in a mixture of two coexisting phases with differing densities $\rho_{1} \neq \rho_{2}$ can be illustrated by a lattice-gas model $[44,45]$.

Let the phases be distinguished by their average densities $\rho_{1}$ and $\rho_{2}$, so that

$$
\rho_{1}>\rho_{2} .
$$

Suppose that the system consists of $N$ particles each of which can be located in one of the $N_{s}$ sites of a crystalline lattice $\{\mathbf{a}\}$, with $i=1,2, \ldots, N_{s}$ and $N \leq N_{s}$. The field operator of a particle in a $\nu$-phase can be presented as an expansion

$$
\psi_{\nu}(\mathbf{r})=\sum_{i n} c_{i n \nu} e_{i \nu} \varphi_{n}\left(\mathbf{r}-\mathbf{a}_{i}\right)
$$

over the Wannier functions $\varphi_{n}\left(\mathbf{r}-\mathbf{a}_{i}\right)$, labelled by a multi-index $n$, with $e_{i \nu}=0,1$ being the occupation variable. Each site can be occupied by not more than one particle, which implies the validity of the unipolarity condition

$$
\sum_{n} c_{i n \nu}^{\dagger} c_{i n \nu}=1
$$

The particles are assumed to be well localized in the lattice sites, so that the matrix elements over the Wannier functions $\varphi_{n}\left(\mathbf{r}-\mathbf{a}_{i}\right)$ for the kinetic operator $\hat{K} \equiv-\hbar^{2} \nabla^{2} / 2 m_{0}$ are

$$
<m i|\hat{K}| n j>=\delta_{m n} \delta_{i j} K_{0}
$$

and for the interaction potential $\Phi\left(\mathbf{r}-\mathbf{r}^{\prime}\right)$ are

$$
<m i, n j|\Phi| m^{\prime} i^{\prime}, n^{\prime} j^{\prime}>=\delta_{m n^{\prime}} \delta_{i j^{\prime}} \delta_{n m^{\prime}} \delta_{j i^{\prime}} \Phi_{i j}
$$

Then the replica Hamiltonian (46) reduces to

$$
H_{\nu}=w_{\nu} \sum_{i}\left(K_{0}-\mu\right) e_{i \nu}+\frac{1}{2} w_{\nu}^{2} \sum_{i \neq j} \Phi_{i j} e_{i \nu} e_{j \nu}
$$

The average phase density (50) takes the form

$$
\rho_{\nu}=\frac{1}{V} \sum_{i}<e_{i \nu}>\text {. }
$$

The chemical potential $\mu$ is defined by the normalization condition (51).

Introducing a quasispin operator

$$
S_{i \nu} \equiv e_{i \nu}-\frac{1}{2}
$$


transforms the replica Hamiltonian (76) to the Ising-type representation

$$
H_{\nu}=E_{\nu}+\frac{1}{2} w_{\nu}^{2} \sum_{i \neq j} \Phi_{i j} S_{i \nu} S_{j \nu}-w_{\nu} \sum_{i} B_{\nu} S_{i \nu}
$$

in which

$$
\begin{gathered}
E_{\nu}=\frac{1}{2} N_{s} w_{\nu}\left(K_{0}-\mu+\frac{1}{4} w_{\nu} \Phi\right), \\
\Phi \equiv \frac{1}{N_{s}} \sum_{i \neq j} \Phi_{i j}, \quad B_{\nu}=\mu-K_{0}-\frac{1}{2} w_{\nu} \Phi .
\end{gathered}
$$

The phase density (77) becomes

$$
\rho_{\nu} \equiv \frac{N_{s}}{2 V}\left(1+m_{\nu}\right)
$$

where

$$
m_{\nu} \equiv \frac{2}{N_{s}} \sum_{i}<S_{i \nu}>
$$

is an analog of a reduced magnetization. Condition (71), owing to Eq. (81), yields

$$
m_{1}>m_{2} \quad\left(\rho_{1}>\rho_{2}\right)
$$

Normalization (51) can be rewritten as the equation

$$
n \equiv \frac{N}{N_{s}}=\frac{1}{2} w_{1}\left(1+m_{1}\right)+\frac{1}{2} w_{2}\left(1+m_{2}\right)
$$

defining the chemical potential $\mu=\mu(n, T)$ as a function of the parameter (84) and temperature $T$.

The considered two-density lattice model can correspond $[13,45]$ to a stable system whose thermodynamic potentials (62) or (63) are lower than the potentials (65) of pure phases, so that

$$
\Omega(T, V, \mu)<\Omega_{\nu}^{\prime}(T, V, \mu), \quad F(T, V, N)<F_{\nu}^{\prime}(T, V, N) .
$$

This means that heterophase turbulence may stabilize condensed matter. That is, turbulent crystals should be rather a rule than an exotic exception. Any real solid can exhibit, under some conditions, especially in the vicinity of transition points, heterophase turbulence, thus, becoming a turbulent crystal.

\section{Ferromagnet with Paramagnetic Fluctuations}

Heterophase fluctuations may arise in systems of different nature. Specific features of the resulting heterophase matter can be well illustrated by a model of ferromagnet with mesoscopic paramagnetic fluctuations [1]. 
The general form of the replica Hamiltonian of a lattice spin system reads

$$
H_{\nu}=w_{\nu} N K_{\nu}+\frac{1}{2} w_{\nu}^{2} N U_{\nu}-w_{\nu}^{2} \sum_{i \neq j} J_{i j \nu} \mathbf{S}_{i \nu} \cdot \mathbf{S}_{j \nu},
$$

where $N$ is the number of spins; $K_{\nu}$, kinetic parameter; $U_{\nu}$, crystalline field; $J_{i j \nu}$, exchange interactions; $\mathbf{S}_{i \nu}$ are spin operators associated with a $\nu$-phase. Ferromagnetic and paramagnetic phases can be distinguished by the order parameter

$$
\eta_{\nu} \equiv\left|<\mathbf{S}_{i \nu}>\right|,
$$

which, in the case of an ideal periodic lattice, does not depend on the lattice-site index. Assume that $\nu=1$ labels the ferromagnetic phase, while $\nu=2$, the paramagnetic phase. By this assumption,

$$
\eta_{1} \neq 1, \quad \eta_{2} \equiv 0
$$

The zero order parameter of the paramagnetic phase supposes that the case without external magnetic fields is considered. In the presence of the latter, the average magnetization of a paramagnet would be nonzero. Then, we could distinguish the phases by the condition $\eta_{1}>\eta_{2}$, similarly to the relations (71) or (83).

Keeping in mind long-range exchange interactions $J_{i j \nu}$, we shall employ the mean-field approximation. The notation

$$
\eta \equiv \eta_{1}, \quad w=w_{1}, \quad J \equiv \frac{1}{N} \sum_{i \neq j} J_{i j 1}
$$

will be used. Then for the probability of ferromagnetic phase, we find

$$
w=\frac{U_{2}+K_{2}-K_{1}}{U_{1}+U_{2}-2 J \eta^{2}},
$$

which follows from Eq. (43) with

$$
\Phi_{\nu}=U_{\nu}-2 J_{\nu} \eta_{\nu}^{2}, \quad J_{\nu} \equiv \frac{1}{N} \sum_{i \neq j} J_{i j \nu} .
$$

For the average spin (87), we have

$$
2 \eta=\tanh \left(w^{2} \frac{J}{T} \eta\right) .
$$

Here and in the following formulas, we shall set $k_{B}=1$. It is reasonable to assume that the arising magnetic order does not influence much the crystalline properties of matter, so that the kinetic terms $K_{\nu}$ and the crystalline fields $U_{\nu}$ do not depend on the phase index $\nu$,

$$
K_{1}=K_{2}, \quad U_{1}=U_{2} \equiv A .
$$

Then, with the notation

$$
u \equiv \frac{A}{J},
$$


the ferromagnetic weight (90) reduces to

$$
w=\frac{u}{2\left(u-\eta^{2}\right)} .
$$

Thus, instead of one equation for the average spin, as it would be in the case of a pure phase, we have now two coupled equations, one for the average spin (91) and another for the probability of ferromagnetic phase (93). Note that the latter can be treated as an additional order parameter that becomes necessary for correctly describing heterophase matter.

The analysis of the model of the heterophase ferromagnet, in the mean-field approximation, shows that there exist two critical temperatures

$$
T_{c}=\frac{1}{8} J, \quad T_{c}^{\prime}=\frac{1}{2} J .
$$

The overall behaviour of the system essentially depends on the value of the dimensionless parameter (92), which measures the relative intensity of disordering interaction $A$ to ordering interaction $J$. According to the stability conditions (44) and (45), paramagnetic fluctuations in a ferromagnet may exist only when $u>\eta^{2}>0$. Therefore, for some $u$ there can exist the nucleation temperature $T_{n}$, defined by the condition

$$
\begin{aligned}
& w \equiv 1 \quad\left(T \leq T_{n}\right) \\
& w<1 \quad\left(T>T_{n}\right) .
\end{aligned}
$$

Depending on the parameter $u$, we can observe five types of behaviour:

(i) $u \leq 0$.

Pure ferromagnetic phase, with $w \equiv 1$, is absolutely stable up to the critical temperature $T_{c}^{\prime}$, where the system becomes paramagnetic. Heterophase ferromagnet can be only metastable, having the critical temperature $T_{c}$.

(ii) $0<u<\frac{3}{2}$.

In the region of temperatures $0 \leq T \leq T_{n}$, the system is a pure ferromagnet, after which paramagnetic fluctuations appear, starting from the nucleation point $T_{n}$, for $T>T_{n}$. The phase transition ferromagnet-paramagnet becomes a first-order transition, occurring at the point $T_{0}$, for which $T_{c}<T_{0}<T_{c}^{\prime}$.

(iii) $0<u<\frac{1}{2}$.

The nucleation point $T_{n}$ exists only for this interval of $u$, such that $0<T_{n}<T_{c}^{\prime}$. When $u \rightarrow 0$, then $T_{n} \rightarrow T_{c}^{\prime}$ and if $u \rightarrow 0.5$, then $T_{n} \rightarrow 0$. For $u>0.5$, there is no nucleation point. The first-order phase transition takes place at $T_{0}$.

(iv) $u=\frac{3}{2}$.

Ferromagnet is always heterophase. The transition temperature $T_{c}$ becomes a tricritical point, where critical indices change by a jump.

(v) $u>\frac{3}{2}$.

Heterophase ferromagnet is stable. The second-order transition happens at the critical temperature $T_{c}$. 
The behaviour of the order parameter, ferromagnetic phase probability, and other thermodynamic characteristics is illustrated in the following figures. The order parameter $\eta_{1}$, defined as the average spin (87), for different values of $u$, is shown in Fig. 4 as a function of $\Theta / J$, with $\Theta=k_{B} T$.

The probability of ferromagnetic phase $w_{1} \equiv w$, satisfying Eq. (93), is presented in Fig. 5 as a function of the reduced temperature $\Theta / J$.

The average magnetization

$$
M \equiv w \eta
$$

as a function of $\Theta / J$ is depicted in Fig. 6. For the values $u$ in the range $-0.25<u<0$, there exists a maximum of the magnetization $M_{m}$ at the temperature $T_{m}$, where

$$
M_{m}=\frac{\sqrt{-u}}{4}, \quad T_{m}=\frac{\sqrt{-u} J}{16 \operatorname{arctanh}(2 \sqrt{-u})} .
$$

These quantities are shown in Fig. 7 as functions of $u \equiv A / J$.

The first-order phase transition to the paramagnetic state occurs at temperature, where the free energy per particle

$$
f \equiv \frac{F}{N}=-\frac{T}{N} \ln \operatorname{Tr} e^{-\tilde{H} / T}
$$

for the heterophase ferromagnet, given by the expression

$$
\frac{f}{J}=\left(w^{2}-w+\frac{1}{2}\right) u+w^{2} \eta^{2}-\frac{T}{J} \ln \left[4 \cosh \left(w^{2} \frac{J}{T} \eta\right)\right],
$$

coincides with the value

$$
\frac{f_{0}}{J}=\frac{u}{4}-\frac{T}{J} \ln 4 .
$$

corresponding to $\eta=0$ and $w=0.5$. Functions (98) and (99) are illustrated in Fig. 8 for $u=0.6$.

The relative entropy

$$
\sigma \equiv \frac{E-F}{N T} \quad(E \equiv<\tilde{H}>)
$$

takes the form

$$
\sigma=-2 w^{2} \frac{J}{T} \eta^{2}+\ln \left[4 \cosh \left(w^{2} \frac{J}{T} \eta\right)\right],
$$

which is depicted in Fig. 9 for different $u$.

The specific heat

$$
C_{H} \equiv \frac{1}{N} \frac{\partial E}{\partial T}=T \frac{\partial \sigma}{\partial T}
$$

in portrayed in Fig. 10, from where it is seen that metastable heterophase ferromagnets, with $u<0$, possess specific-heat maxima below the critical temperature.

When the critical point $T_{c}$ becomes tricritical at $u=3 / 2$, the critical indices change by a jump. Thus, for the critical indices $\alpha$ and $\beta$, characterizing the asymptotic behaviour of the specific heat and magnetization as

$$
C_{H} \sim(-\tau)^{-\alpha}, \quad M \sim(-\tau)^{\beta},
$$


where

$$
\tau \equiv \frac{T-T_{c}}{T_{c}} \rightarrow-0
$$

we obtain

$$
\alpha=\left\{\begin{array}{ll}
0, & u \neq 3 / 2 \\
1 / 2, & u=3 / 2,
\end{array}, \beta= \begin{cases}1 / 2, & u \neq 3 / 2 \\
1 / 4, & u=3 / 2\end{cases}\right.
$$

In any case, $\alpha+2 \beta=1$. If one calculates the critical indices with $\tau$ tending to a small but finite value, instead of $\tau \rightarrow-0$, then one gets the effective indices

$$
\alpha_{e f f}=-\frac{\ln C_{H}}{\ln (-\tau)}, \quad \beta_{e f f}=\frac{\ln M}{\ln (-\tau)} .
$$

These are pictured in Fig. 11 for $|\tau|=10^{-8}$.

Finally, Fig. 12 demonstrates the temperature variation of the average spin, defined by Eq. (91), for different values of the parameter (92). Only the solutions for stable states are presented.

\section{Conclusion}

Mesoscopic heterophase fluctuations may arise in any kind of condensed matter. This is illustrated here for crystals with liquid-like fluctuations, for a two-density lattice mixture, and for ferromagnets with paramagnetic fluctuations. Of course, these fluctuations appear not always, but their existence depends on the system parameters as well as on thermodynamic variables. The presence of such fluctuations can make the system thermodynamically more stable. Their appearance may provoke different pretransitional phenomena and transitional anomalies.

The fluctuations are mesoscopic in size, since they represent the germs of competing thermodynamic phases, which requires that the characteristic length of such a germ be much larger than the mean interparticle distance. At the same time, this characteristic length can be much smaller than the size of the whole sample. If the fluctuations are not static, but evolve in time, appearing and disappearing, their typical lifetimes are to be essentially longer than the local-equilibrium time, but can be much shorter than the observation time, which means that such temporal fluctuations are mesoscopic in time. In any case, these fluctuations are randomly distributed in space.

The spatial randomness of the mesoscopic fluctuations makes it possible to realize the averaging over heterophase configurations and to define an effective renormalized Hamiltonian. In the course of this averaging, novel quantities emerge, representing the probabilities of thermodynamic phases. These probabilities are defined as minimizers of the related thermodynamic potentials.

The theory of heterophase fluctuations [1] provides a general approach for treating a wide class of materials whose properties can be strongly influenced by the existence of such fluctuations. At the same time, many phenomena occurring in condensed matter, which look anomalous without taking account of the mesoscopic fluctuations, can be explained by the influence of the latter. 


\section{References}

[1] V.I. Yukalov, Phys. Rep.. 208, 395 (1991).

[2] M.E. Fisher, Physica A 172, 77 (1991).

[3] Y.A. Alexandrov, Fundamental Properties of Neutron (Clarendon, Oxford, 1992).

[4] A. Gordon, S. Dorfman and D. Fuks, J. Phys. IV 7, 53 (1997).

[5] Y. Yamada et al., Ferroelectrics 240, 363 (2000).

[6] J. Adler and D. Stauffer, Physica A 175, 222 (1991).

[7] K. Mazuruk, C.H. Su, Y.G. Sha and S.L. Lehoczky, J. Appl. Phys. 79, 9080 (1996).

[8] C.H. Su, Y.G. Sha, K. Mazuruk and S.L. Lehoczky, J. Appl. Phys., 80, 137 (1996).

[9] Y.L. Khait, V. Garber, G. Bahir and I. Shapiro, Appl. Phys. Lett. 79, 2990 (2001).

[10] P. Bak, Physica A 163, 403 (1990).

[11] D.E. Feldman, Phys. Rev. Lett. 88, 177202 (2002).

[12] D.E. Feldman, Int. J. Mod. Phys. B 15, 2945 (2001).

[13] V.I. Yukalov and E.P. Yukalova, Int. J. Mod. Phys. B 15, 2433 (2001).

[14] R.H. Parmenter and L.Y. Yu, Chaos 2, 589 (1992).

[15] R.H. Parmenter and L.Y. Yu, Phys. Lett. A 174, 233 (1993).

[16] G.M. Zaslavsky, Phys. Rep. 371, 461 (2002).

[17] J. Volmer, Phys. Rep. 372, 131 (2002).

[18] V.I. Yukalov, Physica A 234, 725 (1997).

[19] V.I. Yukalov, Phys. Rev. E 65, 056118 (2002).

[20] D.P. Kharakoz and E.A. Shlyapnikova, J. Phys. Chem. B 104, 10368 (2000).

[21] V.I. Yukalov, Int. J. Mod. Phys. B 6, 91 (1992).

[22] G. Benedek and K.A. Müller (Eds.), Phase Separation in Cuprate Superconductors (World Scientific, Singapore, 1992).

[23] E. Sigmund and K.A. Müller (Eds.), Phase Separation in Cuprate Superconductors (Springer, Berlin, 1994).

[24] A.J. Coleman, E.P. Yukalova and V.I. Yukalov, Physica C 243, 76 (1995). 
[25] E. Dagotto, T. Hotta and A. Moreo, Phys. Rep. 344, 1 (2001).

[26] Y.L. Khait, Atomic Diffusion in Solids (Scitec, Zurich, 1997).

[27] A.S. Shumovsky and V.I. Yukalov, Physica A 110, 518 (1982).

[28] A.S. Shumovsky and V.I. Yukalov, Chem. Phys. Lett. 117, 617 (1985).

[29] V.I. Yukalov, Chem. Phys. Lett. 229, 239 (1994).

[30] V.I. Yukalov, Physica A 213, 500 (1995).

[31] J.W. Gibbs, Selected Works (Longmans, New York, 1928), Vol. 1.

[32] N.N. Bogolubov, Lectures on Quantum Statistics (Gordon and Breach, New York, 1970), Vol. 2.

[33] V.I. Yukalov, Int. J. Mod. Phys. B 5, 3235 (1991).

[34] A.J. Coleman and V.I. Yukalov, Reduced Density Matrices (Springer, Berlin, 2000).

[35] V.I. Yukalov, Physica A 310, 413 (2002).

[36] V.I. Yukalov, Statistical Green's Functions (Queen's University, Kingston, 1998).

[37] V.I. Yukalov, Phys. Lett. A 81, 433 (1981).

[38] V.I. Yukalov, Phys. Rev. B 32, 436 (1985).

[39] M.V. Jaric (Ed.), Introduction to Quasicrystals (Academic, Boston, 1988).

[40] D. Ruelle, Physica D 7, 40 (1983).

[41] A.C. Newell and Y. Pomeau, J. Phys. A 26, L 429 (1993).

[42] M.Le Berre, E. Ressayre, A. Tallet, Y. Pomeau and L. Di Menza, Phys. Rev. E 66, 026203 (2002).

[43] L.D. Landau and E.M. Lifshitz, Hydrodynamics (Nauka, Moscow, 1986).

[44] V.I. Yukalov, Int. J. Mod. Phys. B 3, 311 (1989).

[45] V.I. Yukalov and E.P. Yukalova, Physica A 213, 485 (1995). 


\section{Figure Captions}

Fig. 1. The crystalline phase probability as a function of temperature.

Fig. 2. The liquid phase probability as a function of temperature.

Fig. 3. The glassy phase probability versus temperature.

Fig. 4. The order parameter $\eta_{1} \equiv \eta$, given by Eq. (91), for different parameters (92): $u=0.5$ (curve 1 ), $u=0.6$ (curve 2 ), $u=0.8$ (curve 3 ), $u=1.5$ (curve 4 ), $u=\infty$ (curve 5 ), $u=-1$ (curve 6$), u=-0.25$ (curve 7 ), $u=-0.1$ (curve 8), $u=-0.01$ (curve 9), $u=-0.001$ (curve 10).

Fig. 5. The ferromagnetic phase probability $w_{1} \equiv w$, defined by Eq. (93), as a function of relative temperature, for the same values of $u$ as in Fig. 4.

Fig. 6. The average magnetization (96) as a function of relative temperature for different values of $u$ as in Fig. 4 .

Fig. 7. The maximum of magnetization $M_{m}$ and the related temperature, as defined in Eq. (97), versus $u \equiv A / J$.

Fig. 8. Free energies per particle, given by Eqs. (98) and (99) for $u=0.6$.

Fig. 9. Relative entropy (101) for the values of $u$ as in Fig. 4.

Fig. 10. Specific heat (102) for different $u$ : $u=0.6$ (curve 2), $u=0.8$ (curve 3), $u=1.5$ (curve 4 ), $u=3$ (curve with no number), $u=\infty$ (curve 5), $u=-1$ (curve 6).

Fig. 11. Effective critical indices (105) for $\tau=-10^{-8}$, versus $u \equiv A / J$.

Fig. 12. Average spin, satisfying Eq. (91), for different values of $u$, which are denoted by the numbers close to the related curves. 